\title{
Complication rate after scoliosis surgery in children with cerebral palsy
}

\author{
Elke Vandendriessche, Marijke Proesmans, Els Ortibus, Pierre Moens
}

From the University Hospitals Leuven, Belgium

Scoliosis is an important problem in children with cerebral palsy (CP). However, the choice for a spinal fusion has to be weighed against the risks of major surgery in this vulnerable population. Paediatricians are frequently consulted preoperatively to assess the (respiratory) risk involved, but data on this question specific for $\mathrm{CP}$ are rare. Therefore, we investigated the complication rate after scoliosis surgery in children with CP, compared to idiopathic scoliosis (IS), and we searched for risk factors for the most common complications. In a retrospective monocenter study we analyzed the medical records from all children with CP and IS undergoing scoliosis surgery from 2010 until 2014. Duration of hospitalization and postoperative complications were compared within a 6-month follow-up. Univariate and multivariate logistic regression models were used to identify risk factors. The study included 44 patients with CP (mean age 15y0mo) and 78 patients with IS (mean age $14 y 6 \mathrm{mo}$ ). Children with $\mathrm{CP}$ experience a higher rate of complications : respiratory and cardiovascular, as well as wound infections and decubitus ulcers $(p<0.05)$. Postoperative pneumonia was the most frequent complication in both patient groups $(43 \%$ and $18 \%$ in $C P$ and IS respectively), with epilepsy being a significant risk factor (OR $3.85, p=0.037$ ) in children with CP. Intensive care unit and total hospital stay were longer in the $\mathrm{CP}$ group $(p<0.001)$. These results may add information on perioperative care and surgical decision making.

Keywords : cerebral palsy ; scoliosis surgery ; complication rate.

P. Moens is co-holder of the SpineVision educational chair: clinical and translational research in the field of paediatric spinal deformity treatment. The authors received no financial support for the research of this article.

\section{INTRODUCTION}

Scoliosis is a common problem in children with cerebral palsy (CP). CP related scoliosis is caused by unequal muscle tone and the risk of developing scoliosis increases with age and is related to the $\mathrm{CP}$ severity, expressed by the Gross Motor Function Classification System (GMFCS) (1,2). At the age of 18 years, $50 \%$ of patients with GMFCS level IV or $\mathrm{V}$ have a clinically moderate to severe scoliosis (2). Scoliosis may impair sitting balance (with risk for decubitus ulcers) causing pain and functional impairment. With regard to the respiratory system, scoliosis decreases chest wall compliance resulting in hypoventilation and restrictive lung disease. Asymmetrical chest expansion leads to unequal lung

Elke Vandendriessche ${ }^{1}, \mathrm{MD}$

- Marijke Proesmans ${ }^{2}, \mathrm{MD}, \mathrm{PhD}$

- Els Ortibus ${ }^{3}, \mathrm{MD}, \mathrm{PhD}$

- Pierre Moens ${ }^{4}, \mathrm{MD}$

${ }^{1}$ Department of Paediatrics, University Hospitals Leuven, Leuven, Belgium.

${ }^{2}$ Department of Paediatric Pulmonology, University Hospitals Leuven, Leuven, Belgium.

${ }^{3}$ Department of Development and Regeneration, University Hospitals Leuven, Leuven, Belgium.

${ }^{4}$ Department of Orthopaedic Surgery, University Hospitals Leuven, Leuven, Belgium.

Correspondence : E. Vandendriessche, Department of Paediatrics, University Hospitals Leuven, Herestraat 49, 3000 Leuven, Belgium. Phone : +32 477404914.

E-mail : evandendriessche.gnk@gmail.com

- 2021, Acta Orthopædica Belgica.

Acta Orthopædica Belgica, Vol. 87 - 2 - 2021 
inflation with ventilation/perfusion mismatch and in rare cases compression of intrathoracic airways secondary to anatomical distortion (1). Because of this negative impact and taking into account that in patients with $\mathrm{CP}$ scoliosis may further progress even after the growth spurt, treatment is essential (1). Bracing is useful in supporting sitting balance and improving overall functioning, however it may only slow the progression of the curve (3-5). Therefore, surgical treatment needs to be considered. However, the choice for a spinal fusion has to be weighed against the risks of major surgery. Many children with $\mathrm{CP}$ have chronic respiratory problems of multifactorial origin. Swallowing disorders and gastroesophageal reflux may give rise to aspiration. Decreased sensitivity of cough receptors and weak breathing muscles lead to ineffective cough and thus airway clearance. In case of bulbar weakness or retrognathia, obstructive breathing may be present especially during sleep. A combination of these risk factors may result in recurrent or chronic airway infections (1). Postoperative pain and immobilization give rise to hypoventilation and decreased cough with risk for atelectasis, pneumonia, difficult weaning and respiratory failure. Lung function tests and expiratory flow measurements may be used for predicting respiratory assessment and for estimating the need for postoperative ventilation, but children with CP can often not adequately perform these measurements.

The risk for postoperative respiratory complications is known to be higher in patients with neuromuscular scoliosis (NMS) compared to patients with idiopathic scoliosis (IS) (6-8). Neuromuscular scoliosis covers a large and heterogeneous group of diagnoses including $\mathrm{CP}$, strict neuromuscular disease such as Duchenne muscular dystrophy but also other neurological, syndromic and metabolic disorders (8-10). Although data are available on the complications after NMS surgery, this is rarely compared to patients with IS or involve a heterogeneous population. Paediatricians are frequently consulted to assess the (respiratory) risk involved before planning this major surgery. Data on this question specific for $\mathrm{CP}$ are rare. Therefore, the aim of this study is to document the complication rate of scoliosis surgery in children with CP compared to patients with IS. Additionally we aim to identify risk factors for the most common complications. With this data we may better inform patients and their caregivers, and we may better anticipate or even prevent complications.

\section{MATERIALS AND METHODS}

Data were collected from all paediatric patients (0-18 years) with CP and IS undergoing scoliosis surgery from 2010 to 2014 in the university hospital of Leuven, Belgium. CP was defined as central motor dysfunction caused by damage to a young, developing brain. Patients with a nonidiopathic scoliosis other than CP were excluded. Patient information was obtained from electronic medical records. All patients underwent surgery by the same orthopaedic surgeon during a singlestaged procedure. There were three types of spinal fusion : posterior, anterior and combined fusions. Instrumentation consisted of pedicle screws for the caudal vertebrae (or iliac screws when fusion reached the pelvis), and hooks and Universal Clamps for fixation of the cranial vertebrae. Antibiotic prophylaxis was routinely administered and included cefazoline, or clindamycin in case of penicillin allergy. After surgery, patients were nursed at the post anesthesia care unit (PACU), or in the intensive care unit (ICU) standard for one night after which they were transferred to the paediatric ward if clinically stable. The study was approved by the hospital's Ethics Committee.

The following demographic and clinical characteristics were retrieved from the medical records: age, gender, weight $\mathrm{z}$-score, feeding method, gastroesophageal reflux disease (GORD), epilepsy, pre-existing respiratory problems, GMFCS level and intellectual disability. The feeding method was divided in full oral feeding versus partial or complete tube feeding (nasogastric tube or percutaneous endoscopic gastrostomy (PEG) tube). GORD was defined as the need for therapy with proton pump inhibitors, a history of Nissen fundoplication or a documented diagnosis of GORD in the patient's medical record. Epilepsy was identified as the need for anti-epileptic drug therapy at time of surgery. Pre-existing respiratory problems were defined as 
one or more pneumonias in the last 5 years, or the need for chronic respiratory medication. Patients were considered to have an intellectual disability when they were unable to attend mainstream school or when they needed special education because of cognitive reasons.

Operative characteristics included the number of fused vertebrae, posterior versus anterior or combined fusions and preoperative Cobb angle on the most recent preoperative spine radiography. In case of an "S-shaped" spinal curve with two angles, only the largest angle was included. The need for perioperative blood transfusion was also evaluated.

The length of ICU hospitalization and total hospital stay, together with all postoperative complications during a 6-month follow-up period were registered. Total hospital stay was defined as sum of days in hospital following surgery excluding eventual readmission. Prolonged ICU hospitalization was defined as sum of days in the ICU excluding the first day following surgery since all children were nursed on the ICU or PACU during one night. Cardiovascular instability was defined as the need for vasopressor agents, or as a systolic blood pressure below $90 \mathrm{mmHg}$ despite intravenous fluid administration. Neurologic complications involved non-transient motor or sensor deficiencies. Delayed extubation was defined as failure of extubation immediately after surgery. Postoperative pneumonia was present if reported so in the patient's medical record.

Statistical analysis was performed by Statistica 12. Study groups were compared using t-test or Mann-Whitney $U$ for continuous variables when appropriate and by $\mathrm{Chi}^{2}$ or Fisher's exact test for discrete variables. Univariate and multivariate logistic regression models were constructed to identify risk factors for postoperative pneumonia. $\mathrm{P}$-values less than 0.05 were considered statistically significant.

\section{RESULTS}

From 2010 until 2014, 206 paediatric patients underwent scoliosis surgery and 78 patients were eligible for inclusion in the IS group and 44 patients in the group with CP (figure 1). Demographic, clinical and operative characteristics are listed in table I.

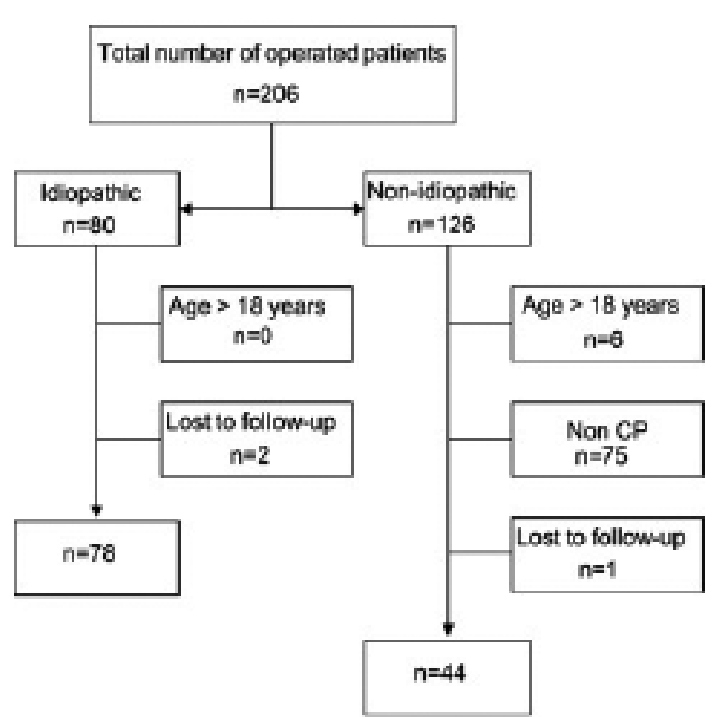

Fig. 1. - Flowchart of patient inclusion. $\mathrm{CP}=$ cerebral palsy.

The majority of patients with $\mathrm{CP}$ were classified in GMFCS level V $(n=31 / 44)$. Only nine patients were classified in GMFCS level IV, one in GMFCS level III and two patients in GMFCS level II. For one patient the GMFCS level was not found. Table II shows the postoperative complications in both the $\mathrm{CP}$ and IS group. The most frequent complication was postoperative pneumonia. Prolonged ICU hospitalization occurred in 24/44 patients with CP versus only $5 / 78$ patients with IS $(p<0.001)$. The median total hospital stay was 11 days (interquartile range (IQR) 9-15) for CP, compared with 8 days (IQR $8-9)$ in the patient group with IS $(p<0.001)$. Other postoperative complications included: cholestasis ( $n=1$ in CP), pancreatitis ( $n=1$ in IS), acute respiratory distress syndrome $(n=1$ in $C P)$, obstructive breathing with need for oxygen therapy, mucolytics and bronchodilatation $(n=1$ in $C P)$, chylothorax with need for clipping of the thoracic duct ( $n=1$ in IS), infection of the gastrostomy wound ( $n=1$ in $C P)$, coagulation disorders for which multiple transfusions with fresh frozen plasma were given $(n=1$ in CP), increase in epileptic seizures $(n=1$ in CP) and increase in spasticity $(n=3$ in $\mathrm{CP}$ ). Univariate and multivariate logistic regression models were constructed to identify risk factors for postoperative pneumonia in children with CP. Results of the univariate analysis are listed in table III. In this analysis the risk for pneumonia in children 
Table I. - Demographic, clinical and operative characteristics

\begin{tabular}{|l|c|c|c|}
\hline & CP $(\mathrm{n}=44)$ & IS $(\mathrm{n}=78)$ & $\mathrm{p}$-value \\
\hline Mean age $(\mathrm{SD})$ & $15 \mathrm{y0mo}(2 \mathrm{y} 0 \mathrm{mo})$ & $14 \mathrm{y} 6 \mathrm{mo}(1 \mathrm{y} 4 \mathrm{mo})$ & 0.048 \\
\hline Gender (M:F) & $24: 20$ & $15: 63$ & $<0.001$ \\
\hline Mean weight z-score (SD) & $-4.09(2.73)$ & $-0.05(1.00)$ & $<0.001$ \\
\hline Mean Cobb angle (SD) & $68(21.82)$ & $58(12.19)$ & 0.017 \\
\hline Mean number of fused vertebrae (SD) & $15(2.64)$ & $13(1.78)$ & $<0.001$ \\
\hline Fusion approach (ASF: PSF: Combined) & $1: 36: 7$ & $0: 72: 5$ & 0.096 \\
\hline Transfusion & 25 & 24 & 0.005 \\
\hline Tube feeding & 17 & 0 & $<0.001$ \\
\hline GORD & 21 & 1 & $<0.001$ \\
\hline Epilepsy & 22 & 0 & $<0.001$ \\
\hline Respiratory problems & 10 & 3 & 0.001 \\
\hline Intellectual disability & 37 & 2 & $<0.001$ \\
\hline
\end{tabular}

a Mean age in years (y) and months (mo). Abbreviations : CP, cerebral palsy ; IS, idiopathic scoliosis ; SD, standard deviation ; ASF, anterior spinal fusion; PSF, posterior spinal fusion; GORD, gastroesophageal reflux disease.

Table II. - Postoperative complications

\begin{tabular}{|l|c|c|c|}
\hline & $\mathrm{CP}(\mathrm{n}=44)$ & IS $(\mathrm{n}=78)$ & $\mathrm{p}$-value \\
\hline Pneumonia & 19 & 14 & 0.003 \\
\hline Atelectasis & 7 & 1 & 0.002 \\
\hline Pleural effusion & 11 & 7 & 0.017 \\
\hline Pneumothorax & 6 & 3 & 0.047 \\
\hline Reintubation & 3 & 0 & 0.020 \\
\hline Delayed extubation & 27 & 2 & $<0.001$ \\
\hline Ileus & 3 & 5 & 0.930 \\
\hline Urinary tract infection & 2 & 2 & 0.556 \\
\hline Urinary retention & 2 & 1 & 0.264 \\
\hline Motor or sensor deficiencies & 1 & 2 & 0.921 \\
\hline Cardiovascular instability & 6 & 1 & 0.005 \\
\hline Septicemia & 2 & 0 & 0.058 \\
\hline Fever of unknown origin & 7 & 7 & 0.248 \\
\hline Wound infection & 6 & 0 & 0.001 \\
\hline Instrumentation failure & 2 & 1 & 0.264 \\
\hline Decubitus ulcer & 8 & 0 & $<0.001$ \\
\hline Mortality & 0 & 0 & $/$ \\
\hline
\end{tabular}

Abbreviations : CP, cerebral palsy; IS, idiopathic scoliosis.

with $\mathrm{CP}$ increased with epilepsy (odds ratio (OR) $3.85, p=0.037)$. There was a tendency for higher pneumonia risk with anterior or combined spinal fusions compared to posterior only (OR of 5.31 with marginal significance $(p=0.060))$ and with GORD (OR of 3.05, $p=0.078$ ). Epilepsy remained a significant risk factor in the multivariate analysis (OR 13.12, $p=0.024$ ). A higher GMFCS level was not found to be a risk factor for postoperative pneumonia, probably because of the small sample size and skewed data. For that reason, the GMFCS level was omitted from the list of risk factors in the multivariate analysis. In the patients with $\mathrm{CP}$ with intellectual disability $(n=37) 51 \%$ had postoperative pneumonia, while in the patients with $\mathrm{CP}$ without intellectual disability $(\mathrm{n}=7)$ no one suffered from postoperative pneumonia $(p=0.014)$. 
Table III - Risk factors for postoperative pneumonia in patients with CP: univariate regression analysis

\begin{tabular}{|c|c|c|c|}
\hline & Odds ratio & $95 \% \mathrm{CI}$ & p-value \\
\hline Age & 0.81 & $0.59-1.10$ & 0.179 \\
\hline Gender $^{\mathrm{a}}$ & 0.54 & $0.16-1.82$ & 0.319 \\
\hline Weight z-score & 1.04 & $0.83-1.29$ & 0.760 \\
\hline Tube feeding & 2.86 & $0.82-10.01$ & 0.101 \\
\hline GORD & 3.05 & $0.88-10.52$ & 0.078 \\
\hline Epilepsy & 3.85 & $1.09-13.66$ & 0.037 \\
\hline Respiratory problems & 2.42 & $0.57-10.25$ & 0.229 \\
\hline Cobb angle & 1.01 & $0.98-1.04$ & 0.358 \\
\hline Type of spinal fusion ${ }^{b}$ & 5.31 & $0.93-30.20$ & 0.060 \\
\hline Number of fused vertebrae & 1.09 & $0.84-1.42$ & 0.495 \\
\hline GMFCS level & 1.02 & $0.45-2.29$ & 0.962 \\
\hline Transfusion & 1.08 & $0.32-3.61$ & 0.900 \\
\hline
\end{tabular}

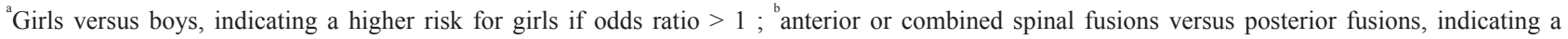
higher risk for anterior or combined spinal fusions if odds ratio $>1$. Abbreviations : CI, confidence interval P. Moens is co-holder of the SpineVision educational chair: clinical and translational research in the field of paediatric spinal deformity treatment. The authors received no financial support for the research of this article. P. Moens is co-holder of the SpineVision educational chair: clinical and translational research in the field of paediatric spinal deformity treatment. The authors received no financial support for the research of this article.; GORD, gastroesophageal reflux disease ; GMFCS, Gross Motor Function Classification System.

\section{DISCUSSION}

Our data confirm that children with CP undergoing spinal fusion have an increased risk for postoperative complications compared to IS patients : respiratory and cardiovascular, as well as wound infections and decubitus ulcers. Postoperative pneumonia was the most frequent complication in both patient groups, with epilepsy being a significant risk factor in children with CP. Because our hospital is a tertiary referral center our study population includes a more severe spectrum of patients with CP compared to other studies which is also reflected by the GMFCS levels. Additionally higher risk patients were not excluded from surgery. The incidence of respiratory complications after scoliosis surgery in NMS patients varies in literature from $2 \%-47 \%$ (7-12). Study population, length of follow-up and definitions of pre and postoperative complications differ between studies. In the meta-analysis of Sharma et al $23 \%$ of NMS patients suffered from respiratory complications, $30 \%$ if only patients with CP were included (11). Samdani et al also reports around $30 \%$ occurrence rate of major pulmonary complications in patients with $\mathrm{CP}$ (13). The incidence of postoperative pneumonia is less reported. In the group of NMS patients postoperative pneumonia was only seen in $4 \%$ to $8 \%$, but this patient group did not have multiple disabilities like our CP cohort (8-10). The study of Nishnianidze et al describing postoperative complications in 303 patients with $\mathrm{CP}$ reports only seven cases of pneumonia, while the study of Nectoux et al diagnosed seven out of 28 patients with $\mathrm{CP}$ with postoperative pneumonia $(14,15)$. The major differences between studies may be caused by different definitions of pneumonia. In our study there is most likely an overestimation of postoperative pneumonia diagnoses, for example if atelectasis with postoperative absorption fever was diagnosed as pneumonia. Moreover our definition did not take into account the severity or clinical impact. In our study 27 of 44 patients with CP were ventilated postoperatively. They could all, except for one patient, be extubated within 24 hours after surgery which makes the contribution of ventilator associated pneumonia less likely. Other studies report longer ventilation times (14-16). In a cohort of 303 patients with CP 154 were ventilated more than 48 hours (14). Although need for reintubation was low in our cohort (three out of 44 patients with $\mathrm{CP}$ ), the former cohort had even lower rates (eight out of 303 patients) (14). In our study the median hospitalization period for patients with $\mathrm{CP}$ was 2 days in ICU and 11 days in total. Other studies report longer total hospitalization and ICU times with total 
hospitalization time between 16 and 18 days and ICU stay up to 6 days $(4,14,16)$. The difference in cardiovascular instability and need for transfusion between CP and IS has been reported in other series. In the cohort of Hod-Feins et al cardiovascular instability (defined as hypotension combined with clinical signs of hypoperfusion) was even reported in six of 21 patients with CP and only in one of 120 IS patients (16). NMS patients have an almost seven times higher risk for extensive blood loss (defined as more than half of the estimated blood volume) compared to IS patients, even after correction for the extent of the spinal fusion (19). Osteopenic bone and decreased venous tone contribute to higher blood loss (19). Our study confirms the rate of wound infections reported in CP spinal surgery ranging between $7 \%$ to $15 \%(14,20,21)$. This high risk can be explained by motor and sensory loss leading to pressure injuries from which infection can spread to the surgical wound directly or via the bloodstream. In case of incontinence, urine or stools can contaminate surgical wounds. In our study no mortality was seen within a follow-up time of 6 months. In a large survey with 19360 paediatric patients undergoing spinal fusion, a mortality rate of $0.3 \%$ in NMS ( $n=4657$ including 1975 patients with CP) and $0.02 \%$ in IS ( $n=11227)$ was registered (12). Mortality within the NMS group was primarily caused by respiratory failure or aspiration (25\%), massive blood loss $(19 \%)$ or sepsis (19\%). Tsirikos et al reported that patients with $\mathrm{CP}$ with prolonged ICU hospitalization stay and patients with severe preoperative thoracic hyperkyphosis experienced the worst long time survival rates (22).

CP patients suffer more from malnourishment, feeding problems (GORD and gastrostomy dependent feeding), epilepsy, respiratory problems and intellectual disability, comorbidities not present in patients with IS. Furthermore patients with CP have a more severe degree of scoliosis with greater Cobb angles and need for extended spinal fusions. All these factors may play a role in the higher risk for postoperative complications $(10,14,23)$. In the multivariate risk factor analysis for our cohort, we found a 13 times higher risk for postoperative pneumonia in patients with epilepsy. In a group of 175 NMS patients (129 CP), there was a significant higher total complication rate in patients with epilepsy (9). The higher pneumonia rate might be explained by aspiration following epileptic seizures. More likely, the epilepsy is an indicator of disease severity within CP. In three other studies no higher complication rate was seen in patients with $\mathrm{CP}$ with epilepsy, but the risk for pneumonia itself was not studied $(13,14,16)$. As can be expected, we found postoperative pneumonia significantly more often in patients with CP with intellectual disability but this has not been reported in other series. In the univariate analysis there was a tendency for higher pneumonia risk in patients with GORD, a known risk factor for (aspiration) pneumonia.

Strengths of our study are the clearly defined study population and a separate risk factor analysis for pneumonia. Since it is a monocenter study perioperative policies are identical for all patients. Limitations of our study are the retrospective character, the limited sample size and the weak definition of pneumonia.

Prevention of respiratory complications include airway clearance techniques and noninvasive ventilation (NIV) but few data are available for patients with CP (17). In a small series NIV was started in 13 patients with NMS of which four were patients with CP (18). No postoperative complications were seen in the patients using NIV. Specifically for the CP group there are no clear risk factors to select the patients eligible for NIV.

In conclusion, we have demonstrated that children with CP undergo spinal fusion with high comorbidity and higher risk for cardiorespiratory and wound complications. Postoperative pneumonia is the most frequent complication with epilepsy being an important risk factor. This information is important in preoperative counselling and decision making. Additional research for NIV and more intensive airway clearance support as support in the postoperative period for a selected group of patients with $\mathrm{CP}$ is necessary.

\section{REFERENCES}

1. Seddon PC, Khan Y. Respiratory problems in children with neurological impairment. Arch Dis Child 2003 ; 88(1) : 75-8. 
2. Persson-Bunke M, Hägglund G, Lauge-Pedersen H, Ma PW, Westbom L. Scoliosis in a total population of children with cerebral palsy. Spine (Phila Pa 1976) 2012 ; 37(12) : 708-13.

3. Jones-Quaidoo SM, Yang S, Arlet V. Surgical management of spinal deformities in cerebral palsy. J Neurosurg Spine 2010 ; 13(6) : 672-85.

4. Sitoula P, Holmes L, Sees J et al. The long-term outcome of early spine fusion for scoliosis in children with cerebral palsy. Clin Spine Surg 2016 ; 29(8) : E406-12.

5. Cloake T, Gardner A. The management of scoliosis in children with cerebral palsy : a review. J Spine Surg 2016 ; 2(4) : 299-309.

6. Divecha HM, Siddique I, Breakwell LM, Millner PA. Complications in spinal deformity surgery in the United Kingdom : 5-year results of the annual British Scoliosis Society National Audit of Morbidity and Mortality. Eur Spine J 2014 ; 23(Suppl. 1) : S55-60.

7. Hod-Feins R, Abu-Kishk I, Eshel G. et al. Risk factors affecting the immediate postoperative course in pediatric scoliosis surgery. Spine (Phila Pa 1976) 2007 Oct; 32(21) : 2355-60.

8. Murphy NA, Firth S, Jorgensen T, Young PC. Spinal surgery in children with idiopathic and neuromuscular scoliosis. What's the difference? J Pediatr Orthop 2006 Mar; 26(2) : 216-20.

9. Mohamad F, Parent S, Pawelek J. et al. Perioperative Complications After Surgical Correction in Neuromuscular Scoliosis. J Pediatr Othop 2007 Jun. 27(4) : 392-7.

10. Master DL, Son-Hing JP, Poe-Kochert C, Armstrong DG, Thompson GH. Risk factors for major complications after surgery for neuromuscular scoliosis. Spine (Phila Pa 1976) 2011 Apr; 36(7) : 564-71.

11. Sharma S, Wu C, Andersen T, Wang Y, Hansen ES. et al. Prevalence of complications in neuromuscular scoliosis surgery : A literature meta-analysis from the past 15 years. Eur Spine J 2013 ; 22(6) : 1230-49.

12. Reames DL, Smith JS, Fu KMG. et al. Complications in the surgical treatment of 19,360 cases of pediatric scoliosis : A review of the scoliosis research society morbidity and mortality database. Spine (Phila Pa 1976) 2011 ; 36(18) : 1484-91.

13. Samdani AF, Belin EJ, Bennett JT. et al. Major perioperative complications after spine surgery in patients with cerebral palsy : assessment of risk factors. Eur Spine J $2016 ; 25(3): 795-800$.

14. Nishnianidze T, Bayhan IA, Abousamra O. et al. Factors predicting postoperative complications following spinal fusions in children with cerebral palsy scoliosis. Eur Spine $J 2016$; 25(2) : 627-34.

15. Nectoux E, Giacomelli MC, Karger C, Herbaux B, Clavert JM. Complications of the Luque-Galveston scoliosis correction technique in paediatric cerebral palsy. Orthop Traumatol Surg Res 2010 ; 96(4) : 354-61.

16. Hod-Feins R, Anekstein Y, Mirovsky Y. et al. Pediatric scoliosis surgery - The association between preoperative risk factors and postoperative complications with emphasis on cerebral palsy children. Neuropediatrics 2007 ; 38(5) : 239-43.

17. Vialle R, Thévenin-Lemoine C, Mary P. Neuromuscular scoliosis. Orthop Traumatol Surg Res 2013 ; 99 (Suppl. 1) : S124-39.

18. Khirani S, Bersanini C, Aubertin G. et al. Non-invasive positive pressure ventilation to facilitate the post-operative respiratory outcome of spine surgery in neuromuscular children. Eur Spine J 2014 ; 23(Suppl. 4) : S406-11.

19. Edler A, Murray DJ, Forbes RB. Blood loss during posterior spinal fusion surgery in patients with neuromuscular disease: Is there an increased risk? Paediatr Anaesth 2003 ; 13(9) : 818-22.

20. Sponseller PD, Shah SA, Abel MF. et al. Infection rate after spine surgery in cerebral palsy is high and impairs results : Multicenter analysis of risk factors and treatment. Clin Orthop Relat Res 2010 ; 468(3) : 711-6.

21. Borkhu B, Borowski A, Shah SA. et al. Antibiotic-loaded allograft decreases the rate of acute deep wound infection after spinal fusion in cerebral palsy. Spine (Phila Pa 1976) $2008 ; 33(21): 2300-4$.

22. Tsirikos AI, Chang WN, Dabney KW, Miller F, Glutting J. Life expectancy in pediatric patients with cerebral palsy and neuromuscular scoliosis who underwent spinal fusion. Dev Med Child Neurol 2003 ; 45(10) : 677-82.

23. Chidambaran V, Gentry C, Ajuba-Iwuji C. et al. A retrospective identification of gastroesophageal reflux disease as a new risk factor for surgical site infection in cerebral palsy patients after spine surgery. Anesth Analg $2013: 117(1): 162-8$. 